Hyperbilirubinaemia

\title{
American Academy of Pediatrics guidelines for detecting neonatal hyperbilirubinaemia and preventing kernicterus
}

\section{Kaplan, C Hammerman}

\section{Are there worldwide implications?}

A lthough neonatal jaundice is usually a self limited condition, in exceptional circumstances, extreme hyperbilirubinaemia, with its devastating potential of irreversible brain damage due to bilirubin encephalopathy or kernicterus, may occur. For the most part, kernicterus should nowadays be a preventable condition; however, cases continue to occur. The reasons for the persistence of kernicterus are undoubtedly multifactorial. One factor may have been an opinion which evolved at the beginning of the 1990s that in some cases higher serum total bilirubin (STB) concentrations could be allowed than were previously acceptable. At about the same time a new phenomenon developed: that of earlier and earlier discharge of the mother-infant dyad. The potential consequences of discharge at or even earlier than 24 hours are that in many instances lactation may not yet be established, jaundice may often not have become manifest, to say nothing of reaching its peak, and associated illnesses may not yet have declared themselves. Additional factors included discharge of borderline premature neonates of 35-37 weeks gestation as if they were born at term, and failure of physicians to abide by the guidelines for the detection of hyperbilirubinaemia and prevention of kernicterus published by the American Academy of Pediatrics (AAP) in 1994. ${ }^{1}$ During the last 15 years, cases of kernicterus were reported not only from the United States, ${ }^{2}$ but also, although to a lesser extent, from other industrialised countries, including Canada, Denmark, Holland, and New Zealand. ${ }^{3}$ The myth that haemolysis was a prerequisite for kernicterus was disproved. ${ }^{4}$ By the year 2001 the number of cases of kernicterus reported was sufficient for the AAP to publish a warning cautioning paediatricians of the potential of kernicterus and the dangers of hyperbilirubinaemia. ${ }^{5}$

In response to the continuing occurrence of cases of kernicterus, the AAP has recently issued a revised set of guidelines. ${ }^{6}$ In this protocol thresholds for starting phototherapy and performing exchange transfusion are lower to an extent than those of the 1994 recommendations. However, the new guidelines are far more comprehensive than simply comprising a manual of when to turn on or off the phototherapy lamps. Rather they offer a wide ranging approach to the detection of hyperbilirubinaemia and the prevention of kernicterus. Granted, as emphasised by the AAP committee members themselves, there is little evidence on which to base many of the AAP's recommendations, and many are based on experience and consensus between the committee members. Some of the salient features of the new guidelines include:

- Strategies for evaluation of the risk of developing hyperbilirubinaemia before discharge.

- Stressing the need to assess the risk of developing hyperbilirubinaemia by plotting the specific STB concentration on the hour of life specific nomogram ${ }^{7}$ : the higher the centile, the greater is the risk of developing hyperbilirubinaemia.

- Emphasising the value, in cases where the STB concentrations are of concern, or rising, of providing intensive phototherapy by putting the infant under multiple banks of phototherapy lights.

- Giving nurses the prerogative of ordering an STB test without requiring a doctor's order.

- Recognising that borderline preterm (35-36 weeks) neonates are at higher risk than term counterparts.

- Guidelines are given for the administration of intravenous immunoglobulin in selected cases of hyperbilirubinaemia due to isoimmunisation.

Some doctors may be concerned that, if the new AAP guidelines are indeed followed, the number of neonates requiring phototherapy or exchange transfusion will increase. Others will find that the implications for change may be minimal. For example, in our practice, from 1994 till the present, we started phototherapy at STB concentrations suggested by the 1994 guidelines for considering phototherapy, rather than mandating it. Thus the upper limit set by the AAP in 2004 for an STB concentration of $428 \mu \mathrm{mol} / \mathrm{l}(25 \mathrm{mg} / \mathrm{dl})$ will not mean, for us, that we will be performing more exchange transfusions or treating more babies under phototherapy. Certainly, although there is room for individualisation, we do feel that the AAP's new cut-off points for starting phototherapy and performing exchange transfusion should probably not be exceeded. Should there be an abundance of risk factors or if clinical judgment deems it necessary, there is no reason not to start phototherapy earlier or at lower concentrations of bilirubin than the AAP recommends.

The guidelines are not limited to the hospital stay. The realisation that in most cases the STB peaks at about 3-5 days, which is often long after the baby has been discharged home, has prompted instructions about when follow up appointments should be scheduled. The timing of follow up was planned to coincide with the projected peak in STB.

To our knowledge, the only countries with nationally applicable, published guidelines are the United States and Canada. ${ }^{68}$ The new AAP guidelines are naturally focused on US medical practice and its interrelationship with the society of that country. The question therefore arises: are the new AAP guidelines relevant only to the United States, or do they have implications for the rest of the world as well? In our opinion, some of the current recommendations are universally applicable, whereas others should be modified or adapted according to the cultural needs or environmental influences of a specific society or geographical area.

We cannot overemphasise the concept of assessing an STB concentration according to the infant's age in hours. ${ }^{8}$ STB concentrations progress dynamically in an upward direction during the first 3-4 days of life. Thus an STB concentration of $188 \mu \mathrm{mol} / \mathrm{l}(11.0 \mathrm{mg} / \mathrm{dl})$ may be of no concern on the third day of life, but may indicate haemolysis, with its potential for severe hyperbilirubinaemia and bilirubin encephalopathy, if it is noted during the first 24 hours of life. Hyperbilirubinaemia is now defined as an STB concentration greater than the 95th centile for hour of life.

Another important AAP recommendation is the assessment of the risk of 
hyperbilirubinaemia before discharge. One method recommended by the AAP includes universal STB determination with assessment of risk according to the hour of life specific nomogram, before discharge. Excellent as this method may be in singling out those neonates who may develop hyperbilirubinaemia after discharge, the method requires a bilirubin test on each and every neonate, many of whom would never develop significant jaundice. An alternative, less invasive option, possibly preferable to the former in countries with limited resources, is the assessment of risk factors for subsequent hyperbilirubinaemia, with actual bilirubin testing reserved for those who do become jaundiced. Risk factors for every neonate should be assessed before discharge: the greater the number of risk factors, the greater the risk of developing hyperbilirubinaemia.

The AAP also emphasises the need to establish successful lactation as a preventive measure against the development of neonatal hyperbilirubinaemia and suggests indicators of successful nutrition and hydration to be monitored. Although it is important to ensure that lactation has been successfully established, this does not necessarily mean that in each and every instance the dyad should remain in hospital until this goal is reached. In societies that provide a visiting nurse service, the nurse may be able to continue the lactation learning process successfully in the comfort of the home while at the same time following up the baby for hyperbilirubinaemia.

When necessary, and especially in cases in which STB concentrations are continuing to rise despite treatment or are approaching the indications for exchange transfusion, phototherapy should be administered as effectively as possible. Several banks of lights may be used, and fluorescent lamps may be placed close to the baby so as to increase the light irradiance intensity. ${ }^{9}$ We concur that, in most cases of babies readmitted for breast feeding jaundice, in accordance with AAP recommendations, there is no need to discontinue breast feeding. We believe and agree with the AAP that the nurses managing the baby should be allowed to send blood for an STB determination without the delay of obtaining a doctor's order.

On the other hand, some recommendations should be customised and tailored according to the individual character of different countries and varying population groups. For example, selective, rather than universal, screening before discharge may be an option in high risk cases such as glucose-6-phosphate dehydrogenase deficiency, early discharge by parental request, or infants $\leqslant 37$ weeks gestation. Depending on the follow up services available, parental reliability, distances to be travelled in order to obtain medical services, and availability of these services during weekends or holidays, some high risk neonates may benefit from delayed discharge from hospital. Others may be safely discharged if facilities exist for a home visit from a nurse trained in the care of the newborn, when supportive laboratory and medical services can be called on when necessary.

Cultural differences between population groups may either exacerbate jaundice or facilitate identification of jaundice. Thus feeding the baby herbal teas or applying certain umbilical potions or henna applications to the skin may precipitate haemolysis in population groups with a high incidence of glucose-6-phosphate dehydrogenase deficiency. On the other hand, a routine visit of a Jewish ritual circumciser (Mohel) may serve to discover a jaundiced baby in whom the condition was not recognised by the parents.

In countries where formulation of a set of recommendations is envisaged, it may be wise for bilirubin experts of those countries to base their recommendations on the AAP guidelines, while taking into account individualised, region specific factors appropriate to the cultural variations and needs of the specific population groups under their care. It must be appreciated that no set of instructions can be all encompassing and cover each and every possibility. All those involved in the care of the neonate should study the new recommendations. Their use, in combination with individualisation, evaluation of risk factors, and application of common sense, should limit the number of cases of severe hyperbilirubinaemia and kernicterus to the minimum.

Arch Dis Child Fetal Neonatal

Ed 2005;90:F448-F449.

doi: 10.1136/adc.2004.068726

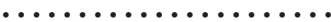

Authors' affiliations

M Kaplan, Department of Neonatology, Shaare Zedek Medical Center and Faculty of Medicine of the Hebrew University, Jerusalem, Israel

C Hammerman, Faculty of Health Sciences, Ben Gurion University of the Negev, Be'er Sheva, Israel

Correspondence to: Dr Kaplan, Department of Neonatology, Shaare Zedek Medical Center, PO Box 3235, Jerusalem 91031, Israel; kaplan@cc.huji.ac.il

Competing interests: none declared

\section{REFERENCES}

1 American Academy of Pediatrics. Practice parameter: management of hyperbilirubinemia in the healthy term newborn. Pediatrics 1994;94:558-65.

2 Johnson LH, Bhutani VK, Brown AK. Systembased approach to management of neonatal jaundice and prevention of kernicterus. J Pediatr 2002;140:396-403.

3 Kaplan M, Hammerman C. Understanding and preventing severe hyperbilirubinemia: is bilirubin neurotoxicity really a problem in the developed world? Clin Perinatol 2004;31:555-75.

4 Maisels MJ, Newman TB. Kernicterus in otherwise healthy, breast-fed term newborns. Pediatrics 1995; $96: 730-3$

5 American Academy of Pediatrics, Subcommittee on Neonatal Hyperbilirubinemia. Neonatal jaundice and kernicterus. Pediatrics 2001; 108:763-5.

6 American Academy of Pediatrics, Subcommittee on Hyperbilirubinemia. Management of hyperbilirubinemia in the newborn infant 35 or more weeks of gestation. Pediatrics 2004; 114:297-316.

7 Bhutani VK, Johnson L, Sivieri EM. Predictive ability of a predischarge hour-specific serum bilirubin for subsequent significant hyperbilirubinemia in healthy term and near-term newborns. Pediatrics 1999; 103:6-14.

8 Fetus and Newborn Committee, Canadian Paediatric Society. Approach to the management of hyperbilirubinemia in term newborn infants. Paediatr Child Health 1999;4:161-4.

9 Maisels MJ. Why use homeopathic doses of phototherapy? Pediatrics 1996;98:283-7. 


\section{American Academy of Pediatrics guidelines for detecting neonatal hyperbilirubinaemia and preventing kernicterus}

\section{Manning}

\section{Are they applicable in Britain?}

n 2004 the American Academy of Pediatrics (AAP) revised its guidelines $I$ for management of severe hyperbilirubinaemia in the newborn. ${ }^{1}$ The objective was to strike a balance between preventing severe hyperbilirubinaemia and its sequelae on the one hand and minimising overinvestigation and treatment of physiological jaundice on the other.

\section{BACKGROUND TO THE REVISION OF THE 1994 GUIDELINES}

The previous guidelines, published in 1994, ${ }^{2}$ reflected a "kinder, gentler" approach to neonatal jaundice. ${ }^{3}$ Thanks to improvements in the management of rhesus isoimmunisation, bilirubin encephalopathy and kernicterus had virtually disappeared among term infants in the western world. Many paediatricians were concerned that this experience was extrapolated inappropriately to neonatal jaundice in general, and that infants with physiological and breast milk jaundice were undergoing unnecessary investigation and treatment. The 1994 guidelines were directed to reducing such intervention. They included the recommendation, however, that all infants discharged within 48 hours of birth should be followed up within three days.

Increasingly early postnatal discharge of term and near term infants, and initiatives to promote breast feeding, coincided with the more relaxed approach to neonatal jaundice. Ironically, these developments may have contributed to the re-emergence of severe jaundice and bilirubin encephalopathy in North America and Europe. ${ }^{4-6}$ Reported associations included relatively short gestation ${ }^{7}$ and comorbidity, particularly glucose-6-phosphate dehydrogenase deficiency. ${ }^{4}$ Possibly early discharge, before jaundice has reached a maximum and breast feeding is established, leaves some infants, particularly if immature or ill, vulnerable to severe jaundice, which would previously have been identified during inpatient postnatal stay. Non-white infants may be particularly vulnerable because, compared with white infants, jaundice is more difficult to evaluate clinically and glucose-6-phosphate dehydrogenase deficiency is more common.

The pendulum thus swung away from the "kinder, gentler" approach and led to the recent revision of the AAP guidelines. The experts who revised them acknowledged the dearth of evidence to underpin their recommendations. The general objectives were to promote breast feeding, to encourage assessment before discharge of the risk of developing later jaundice, to individualise follow up arrangements, and to review treatment advice.

\section{HOW DO THE NEW GUIDELINES DIFFER FROM THE 1994 VERSION?}

One of the most important changes is to extend the scope from full term infants to those of 35 weeks gestation or greater. This change acknowledges that near term infants are being discharged earlier, and may be particularly vulnerable to jaundice because of relative feeding difficulty and hepatic immaturity.

Another substantial change is the inclusion of rather complex recommendations on risk assessment before discharge for severe jaundice, and targeted follow up based on this after discharge. Risk assessment uses a nomogram derived from measurements before discharge of serum bilirubin in 2840 term and near term infants in Philadelphia. ${ }^{8}$ These measurements were reported to predict later significant hyperbilirubinaemia. Two options are proposed for risk assessment: (a) universal total serum bilirubin measurement; $(b)$ bilirubin measurement combined with assessment for clinical risk factors. The timing of the recommended follow up depends on the timing of discharge and the risk assessment before discharge. All infants should be examined by a health professional within a few days of discharge, and those discharged within 24 hours should be reviewed by 72 hours. Infants with risk factors should be reviewed earlier and more often than those without. The recommended location of follow up is not specified but, according to the 1994 guidelines, might be the paediatrician's office, a community clinic, or the family home.

Acknowledging that visual assessment for jaundice severity can be unreliable, the report reviews research in transcutaneous bilirubinometry and recommends that this be evaluated further.

Finally, the guidelines give advice on treatment of moderate to severe jaundice and bilirubin encephalopathy. The phototherapy and exchange transfusion bilirubin thresholds for well infants are slightly lower than in the 1994 guidelines, and even lower thresholds are recommended for infants who are ill or have risk factors for severe jaundice.

\section{ARE THE NEW GUIDELINES APPLICABLE TO NEONATAL PRACTICE IN BRITAIN?}

To address this question, we need to know whether severe jaundice is reemerging in the United Kingdom. The only recent published information is from Wirral, where the incidence of moderate jaundice (serum bilirubin $\geqslant 340 \mu \mathrm{mol} / \mathrm{l}$ ) increased from $2.4 / 1000$ live births in 1991 to $5.5 / 1000$ in 2001 $(p<0.0001) .{ }^{9}$ This increase coincided with a progressive reduction in length of postnatal stay for mothers and infants.

Because of the dearth of British data, a prospective study, supported by the British Paediatric Surveillance Unit, to determine the incidence of severe neonatal jaundice (unconjugated serum bilirubin $\geqslant 510 \mu \mathrm{mol} / \mathrm{l}$ in the first month of life) in Britain and Ireland is in progress. Secondary objectives are to document associated clinical and demographic variables and short term outcomes. Surveillance started in June 2003 and finished in May 2005. Early returns indicate that severe jaundice and bilirubin encephalopathy, although uncommon, are occurring in Britain and Ireland. Associations are similar to those reported in North America: relative immaturity, early discharge, breast feeding, ethnic minority origin, and comorbidity such as haemolysis and sepsis. Raising awareness of these associations therefore is just as relevant here as in North America.

The aim of the guidelines to support breast feeding is laudable, but the specific recommendations-advising mothers to feed their infants 8-12 times 
a day and advising against routine supplementation with water and dextrose-are accompanied by little detail on appropriate support. In Britain, this support is provided by the midwife who, by continuing care after hospital discharge, bridges the gap between secondary and primary care of mother and infant. The AAP recommendations on supporting lactation have little to add to current British practice.

Given the lack of evidence to support specific bilirubin threshold levels for phototherapy and exchange transfusion, the recommendations on treatment of moderate and severe jaundice seem reasonable and do not differ greatly from British practice. The principle of recommending lower thresholds for ill or vulnerable infants is also reasonable, as is the promotion of further research in transcutaneous bilirubinometry.

The most controversial recommendations, and those that are arguably least applicable here, are those pertaining to individualised risk assessment and targeted follow up. Even if early bilirubin measurements were sufficiently predictive of risk, arguably a nomogram would have to be devised for each population of infants to which it applied, reflecting the population incidence and severity of hyperbilirubinaemia, the uptake of breast feeding, and the incidence of haemolytic disease due to blood group incompatibility and glucose-6-phosphate dehydrogenase deficiency.

I am concerned, moreover, that risk assessment and follow up based largely on bilirubin concentrations may be insufficient safely to identify infants at risk for severe hyperbilirubinaemia. Although the guidelines rightly draw attention to other relevant clinical and demographic risk factors, these are too common to be sufficiently predictive of risk. Individualised frequency and timing of follow up arrangements may be too complicated to be implemented effectively. Indeed, this may be the American experience; of 61 infants known to the Pilot Kernicterus Registry who were readmitted from home with severe jaundice, follow up according to the 1994 guidelines had not been arranged in 44 cases. ${ }^{4}$
Appointments had been arranged for a further three infants, but had been missed. This system failure may have indicated complacency about severe jaundice, but probably also reflected the difficulties of implementing individualised follow up programmes.

Currently in the United Kingdom, all mothers and infants receive regular postnatal home visits from their midwives. ${ }^{10}$ Although practice may vary by district, usually the first visit takes place on the day after hospital discharge. Although there is no evidence that this practice is superior to targeted surveillance, ${ }^{10}$ the British system may be more effective than the American in the identification and timely management of severe jaundice. This can progress rapidly in haemolytic disease and in breast feeding failure complicated by dehydration, and so may not always be predicted by bilirubin concentration before discharge nor intercepted by interval follow up. Also, the midwife's visits serve more purpose than visual assessment for jaundice. They offer emotional and practical support to the mother, and permit assessment of the infant's state of hydration and general wellbeing. There is, indeed, some evidence to suggest that home visiting is effective in promoting successful breast feeding. ${ }^{10}$ It may also offer better access to health professionals, particularly for poor and isolated families, than systems that require the infant to be taken to a clinic or office and which put the onus on the family to seek help if the infant is unwell.

Although British postnatal home visiting has been subjected to little evaluation, a recent American study compared the cost effectiveness of universal follow up with review based on bilirubin concentrations before discharge, and estimated that the former was more expensive. ${ }^{11}$ The authors acknowledged, however, that universal follow up may have benefits other than surveillance for jaundice, so its overall cost effectiveness is likely to be greater than their estimates. Pending further evidence, I see no reason for British practice to change to the American model of targeted surveillance for severe jaundice.

\section{CONCLUSIONS}

The revised AAP guidelines are valuable in combating complacency about neonatal jaundice, in highlighting clinical and demographic factors that demand vigilance, and in informing treatment. All of these are relevant to British neonatal care. The case has not been made, however, to abandon the British system of midwifery based universal surveillance of recently discharged infants in favour of risk assessment and targeted surveillance.

Arch Dis Child Fetal Neonatal Ed 2005;90:F450-F451.

doi: 10.1136/adc. 2004.070375

Correspondence to: Dr Manning, Wirral Hospital, Arrowe Park, Wirral, Merseyside CH49 5PE, UK; donal.manning@whnt.nhs.uk

Competing interests: none declared

\section{REFERENCES}

1 American Academy of Pediatrics. Clinical practice guideline: management of hyperbilirubinaemia in the newborn infant 35 or more weeks of gestation. Pediatrics 2004; 114:297-316.

2 American Academy of Pediatrics. Practice parameter: management of hyperbilirubinaemia in the healthy term newborn. Pediatrics 1994;94:558-65.

3 Newman TB, Maisels MJ. Evaluation and treatment of jaundice in the term newborn: a kinder, gentler approach. Pediatrics 1992:89:809-18.

4 Johnson LH, Bhutani VK, Brown AK. Systembased approach to management of neonatal jaundice and prevention of kernicterus. J Pediatr 2002; 140:396-403.

5 Ebbesen F. Recurrence of kernicterus in term and near-term infants in Denmark. Acta Paediatr 2000;89:1213-17.

6 Ebbesen F, Andersson C, Verder H, et al. Extreme hyoerbilirubinaemia in term and near-term infants in Denmark. Acta Paediatr 2005:94:59-64

7 Newman TB, Liljestrand P, Escobar GJ. Infants with bilirubin levels of $30 \mathrm{mg} / \mathrm{dL}$ or more in a large managed care organisation. Pediatrics 2003:111:1303-11.

8 Bhutani VK, Johnson L, Sivieri EM. Predictive ability of a predischarge hour-specific bilirubin for subsequent significant hyperbilirubinaemia in healthy term and near-term infants. Pediatrics 1999:103:6-14

9 Walston F, Manning D, Neithercut WD. Increasing incidence of moderate hyperbilirubinaemia in Wirral. Arch Dis Child Fetal Neonatal Ed 2004;89:374.

10 Bull J, McCormick G, Swann C, et al. Ante- and post-natal home-visiting programmes: a review of reviews. Health Development Agency 2004 www.hda.nhs.uk/evidence.

11 Suresh GK Clark RE. Cost-effectiveness of strategies that are intended to prevent kernicterus in newborn infants. Pediatrics

2004; 114:917-24. 